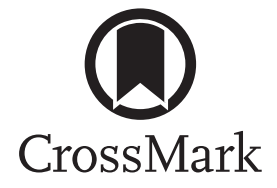

\section{Pulmonary rehabilitation for patients with COPD during and after an exacerbation-related hospitalisation: back to the future?}

To the Editor:

The European Respiratory Society (ERS) and American Thoracic Society (ATS) guideline on management of chronic obstructive pulmonary disease (COPD) exacerbations was published in the March 2017 issue of the European Respiratory Journal [1]. Based on evidence syntheses, including meta-analyses, relevant evidence up to September 2015 was summarised and clinical recommendations for treatment of COPD exacerbations were formulated. These guidelines were endorsed by the ERS Executive Committee and approved by the ATS Board of Directors in December 2016.

These guidelines should provide the basis for rational decisions in the treatment of COPD exacerbations. Unexpectedly, however, this ERS/ATS Task Force made a conditional recommendation against the initiation of pulmonary rehabilitation during hospitalisation and a conditional recommendation in favour of starting pulmonary rehabilitation $\leqslant 3$ weeks after hospital discharge. Moreover, the Task Force rated the quality of evidence for both conditional recommendations to be very low. We take the liberty to challenge these conditional recommendations as well as the assessment of the evidence relating to them. Indeed, there are multiple arguments to recommend the initiation of pulmonary rehabilitation during and (especially) directly after hospitalisation.

Patients with COPD report a broad spectrum of symptoms during exacerbations, including dyspnoea, depression and fatigue [2], which seem to be the basis of the extreme declines in physical activity during hospitalisations [3]. Additionally, comorbidities may flare up [4], and significant losses in quadriceps muscle strength [5], exercise tolerance [6] and health status occur during hospitalisation [7], which only partially recover with usual care after discharge $[3,5,8]$. In addition, it has been shown that patients with anxiety and depression have a doubled risk of short-term hospital re-admission [9]. Therefore, we strongly believe that there is a clear rationale to start early rehabilitative interventions during exacerbation-related hospitalisation of patients with COPD and to continue this after discharge. Importantly, the content of the integrated peri-exacerbation pulmonary rehabilitation programme (which is much more than physiotherapy alone) needs to be tailored to the patient's physical and psychological status [10].

Recent randomised controlled trials (RCTs) have shown that rehabilitative interventions initiated during patients' hospital stay prevent a decline in lower-limb muscle function, balance and exercise performance, and facilitates recovery afterwards [11-15]. These RCTs did not report serious adverse events. Starting early rehabilitation in the hospital setting is clearly also in line with international developments to encourage early rehabilitative interventions, which are safe and effective for patients with a spectrum of illnesses, even in mechanically ventilated, critically ill patients [16-19].

The current ERS/ATS Task Force concluded that pulmonary rehabilitation initiated during hospitalisation increased mortality [1]. This conclusion seems based solely on the study by GREENING et al. [20], who reported a significant difference in mortality at 12 months between the rehabilitative group (starting with a median of three rehabilitative sessions during a median 5-day hospital stay, followed by a 6-week unsupervised home-based programme supported by telephone consultations) and the usual care control group (who did receive daily physiotherapy during hospitalisation as per standard UK practice). As argued

@ERSpublications

Healthcare professionals should educate COPD patients and recommend rehabilitation in the periexacerbation period http://ow.ly/gaiC30eQlVE

Cite this article as: Spruit MA, Singh SJ, Rochester CL, et al. Pulmonary rehabilitation for patients with COPD during and after an exacerbation-related hospitalisation: back to the future? Eur Respir J 2018; 51: 1701312 [https://doi.org/10.1183/13993003.01312-2017]. 
previously [21], the difference in mortality began $>5$ months after the completion of the intervention. While of concern, the difference does not clearly relate to the early rehabilitation intervention. Indeed, the per protocol analysis did not show a difference in mortality, suggesting that those who actually received the intervention were not those who came to harm [20]. Thus, it is questionable to base a conditional recommendation against the initiation of pulmonary rehabilitation during hospitalisation on a single trial and not on the entirety of evidence. Also, it is not entirely clear how the quality of the evidence relating to this recommendation was rated by the ERS/ATS Task Force [1], which led to very different conclusions on the quality of the evidence compared to the recently updated Cochrane review [22].

A conditional recommendation indicates that well-informed patients may make different choices regarding whether or not to have the intervention [1]. While such preference-sensitive decision making is most welcome, it should be noted that, unfortunately, patients with COPD are mostly unaware of the value of (peri-exacerbation) pulmonary rehabilitation programmes for their health outcomes [23]. Moreover, our anecdotal clinical experience shows that healthcare professionals who are uneducated in pulmonary rehabilitation are already using this ERS/ATS conditional recommendation to withhold an early rehabilitative intervention in the peri-exacerbation period from patients. Indeed, during the ERS School Course on Pulmonary Rehabilitation (April 2017 in Athens, Greece), multiple concerns were expressed by participants that their local physicians read the ERS/ATS guideline on management of COPD exacerbations and, in turn, did not refer for early pulmonary rehabilitation.

On December 8, 2016, Puhan et al. [22] published an update of their Cochrane review entitled "Pulmonary rehabilitation following exacerbations of chronic obstructive pulmonary disease", including 20 studies, and concluded that "Quality of life and exercise capacity were improved by rehabilitation, that the effect was substantially larger than the minimal important difference, and the quality of evidence according to GRADE was high. Results for hospital readmissions and mortality were diverse, with some studies showing that pulmonary rehabilitation reduced hospital admissions and mortality compared with usual community care (no rehabilitation), and other studies not showing such effects". Based on the existing literature, we believe that the ERS/ATS Task Force should have made a strong recommendation in favour of starting pulmonary rehabilitation in the first weeks after hospital discharge, without the conditional reservation.

To conclude, pulmonary rehabilitation ambassadors around the world have promoted pulmonary rehabilitation in patients with COPD during and shortly after an exacerbation-related hospitalisation [10, 24], as this results in clinically relevant improvements in exercise performance, lower-limb muscle function, balance and quality of life compared to usual care [22]. Presently, $>75 \%$ of current pulmonary rehabilitation programmes include those patients [25]. Obviously, we recognise the heterogeneity of effects on mortality and hospital re-admission, which should be monitored closely. As always, the specific content of the rehabilitation intervention in the peri-exacerbation period must be tailored carefully to the patient's condition and needs. Nevertheless, given the current evidence, the basis for the ERS/ATS conditional recommendation against the initiation of pulmonary rehabilitation during hospitalisation is not clear. This recommendation is therefore likely to have adverse impact on the quality of care, and in turn, the physical and emotional function and quality of life of these patients. We believe this recommendation is potentially harmful for the further broadening of the scope of pulmonary rehabilitation and sets pulmonary rehabilitation back 15 years. Therefore, we would like to encourage healthcare professionals to educate their patients and recommend pulmonary rehabilitation in the peri-exacerbation period, as only $5-15 \%$ of hospitalised COPD patients are currently referred for early pulmonary rehabilitation [26]. The ERS and ATS should commit to undertake actions that will improve access to and pulmonary rehabilitation services for suitable patients, including those with an exacerbation-related hospitalisation [24].

Martijn A. Spruit $\oplus^{1,2,3}$, Sally J. Singh ${ }^{4,5}$, Carolyn L. Rochester ${ }^{6,7}$, Neil J. Greening ${ }^{8}$, Frits M.E. Franssen $\oplus^{1,2}$, Fabio Pitta, ${ }^{9}$ Thierry Troosters $^{10}$, Claire Nolan ${ }^{11,12}$, Ioannis Vogiatzis ${ }^{13}$, Enrico M. Clini $\odot^{14}$, William D-C. Man ${ }^{11,12}$, Chris Burtin ${ }^{3}$, Roger S. Goldstein $\oplus^{15}$, Lowie E.G.W. Vanfleteren ${ }^{1,2}$, Klaus Kenn ${ }^{16,17}$, Linda Nici ${ }^{18,19}$, Daisy J.A. Janssen ${ }^{1,20}$, Richard Casaburi ${ }^{21}$, Takanobu Shioya ${ }^{22}$, Chris Garvey ${ }^{23}$, Brian W. Carlin ${ }^{24}$, Richard L. ZuWallack ${ }^{25}$, Michael Steiner ${ }^{26}$, Emiel F.M. Wouters ${ }^{1,27}$ and Milo A. Puhan ${ }^{28}$

${ }^{1}$ Dept of Research and Education, CIRO+, Center of Expertise for Chronic Organ Failure, Horn, The Netherlands. ${ }^{2}$ Dept of Respiratory Medicine, Maastricht University Medical Centre, NUTRIM School of Nutrition and Translational Research in Metabolism, Maastricht, The Netherlands. ${ }^{3}$ REVAL - Rehabilitation Research Center, BIOMED Biomedical Research Institute, Faculty of Medicine and Life Sciences, Hasselt University, Diepenbeek, Belgium. ${ }^{4}$ Centre for Exercise and Rehabilitation Science, University Hospitals of Leicester NHS Trust, Glenfield Hospital, Leicester, UK. ${ }^{5}$ School of Sport, Exercise and Health Sciences, Loughborough University, Loughborough, UK. ${ }^{6}$ Yale University School of Medicine, New Haven, CT, USA. ${ }^{7}$ VA Connecticut Healthcare System, West Haven, CT, USA. ${ }^{8}$ Centre for Exercise and Rehabilitation Medicine, University Hospitals of Leicester, Leicester, UK. ${ }^{9}$ Laboratory of Research in Respiratory Physiotherapy, State University of Londrina, Londrina, Brazil. ${ }^{10} \mathrm{KU}$ Leuven, Dept of Rehabilitation Sciences, Leuven, Belgium. ${ }^{11}$ Harefield Pulmonary Rehabilitation and Muscle Research Laboratory, Royal Brompton and Harefield NHS Foundation Trust, London, UK. ${ }^{12}$ National Heart and Lung Institute, Imperial College London, London, UK. ${ }^{13}$ Dept of 
Sport, Exercise and Rehabilitation, Northumbria University, Newcastle Upon Tyne, UK. ${ }^{14}$ University of Modena and Reggio Emilia, Dept of Medical and Surgical Sciences, Modena, Italy. ${ }^{15}$ Dept of Respiratory Medicine and Rehabilitation, University of Toronto, Ontario, Canada. ${ }^{16}$ Schoen Klinik Berchtesgadener Land, Schoenau, Germany. ${ }^{17}$ University of Marburg, Marburg, Germany. ${ }^{18}$ Providence VAMC, Providence, RI, USA. ${ }^{19}$ Brown University, Providence, RI, USA.

${ }^{20}$ Centre of Expertise for Palliative Care, Maastricht UMC+, Maastricht, The Netherlands. ${ }^{21}$ Rehabilitation Clinical Trials Center, Los Angeles Biomedical Research Institute at Harbor-UCLA Medical Center, Torrance, CA, USA. ${ }^{22}$ Dept of Physical Therapy, Akita University Graduate School of Health Sciences, Akita, Japan. ${ }^{23}$ Pulmonary Rehabilitation and Sleep Medicine, University of California San Francisco, CA, USA. ${ }^{24}$ Sleep Medicine and Lung Health Consultants, Pittsburgh, PA, USA. ${ }^{25}$ St Francis Hospital Medical Center, Hartford, CT, USA. ${ }^{26}$ Leicester Respiratory Biomedical Research Centre, Glenfield Hospital, Leicester, UK. ${ }^{27}$ Dept of Respiratory Medicine, Maastricht UMC+, Maastricht, the Netherlands. ${ }^{28}$ Epidemiology, Biostatistics and Prevention Institute, University of Zurich, Zurich, Switzerland.

Correspondence: Martijn A. Spruit, Dept of Research and Education, CIRO, Hornerheide 1, 6085 NM Horn, The Netherlands. E-mail: martijnspruit@ciro-horn.nl

Received: July 052017 | Accepted: Aug 082017

Conflict of interest: Disclosures can be found alongside this article at erj.ersjournals.com

\section{References}

1 Wedzicha JA, Miravitlles M, Hurst JR, et al. Management of COPD exacerbations: a European Respiratory Society/American Thoracic Society guideline. Eur Respir J 2017; 49: 1600791.

2 Kessler R, Stahl E, Vogelmeier C, et al. Patient understanding, detection, and experience of COPD exacerbations: an observational, interview-based study. Chest 2006; 130: 133-142.

3 Pitta F, Troosters T, Probst VS, et al. Physical activity and hospitalization for exacerbation of COPD. Chest 2006; 129: 536-544.

4 Burt MG, Roberts GW, Aguilar-Loza NR, et al. Relationship between glycaemia and length of hospital stay during an acute exacerbation of chronic obstructive pulmonary disease. Intern Med J 2013; 43: 721-724.

5 Spruit MA, Gosselink R, Troosters T, et al. Muscle force during an acute exacerbation in hospitalised patients with COPD and its relationship with CXCL8 and IGF-I. Thorax 2003; 58: 752-756.

6 Carr SJ, Hill K, Brooks D, et al. Pulmonary rehabilitation after acute exacerbation of chronic obstructive pulmonary disease in patients who previously completed a pulmonary rehabilitation program. J Cardiopulm Rehabil Prev 2009; 29: 318-324.

7 Miravitlles M, Ferrer M, Pont A, et al. Effect of exacerbations on quality of life in patients with chronic obstructive pulmonary disease: a 2 year follow up study. Thorax 2004; 59: 387-395.

8 Donaldson GC, Wilkinson TM, Hurst JR, et al. Exacerbations and time spent outdoors in chronic obstructive pulmonary disease. Am J Respir Crit Care Med 2005; 171: 446-452.

9 Dahlen I, Janson C. Anxiety and depression are related to the outcome of emergency treatment in patients with obstructive pulmonary disease. Chest 2002; 122: 1633-1637.

10 Spruit MA, Singh SJ, Garvey C, et al. An official American Thoracic Society/European Respiratory Society statement: key concepts and advances in pulmonary rehabilitation. Am J Respir Crit Care Med 2013; 188: e13-e64.

11 Troosters T, Probst VS, Crul T, et al. Resistance training prevents deterioration in quadriceps muscle function during acute exacerbations of chronic obstructive pulmonary disease. Am J Respir Crit Care Med 2010; 181: 1072-1077.

12 Torres-Sanchez I, Valenza MC, Cabrera-Martos I, et al. Effects of an exercise intervention in frail older patients with chronic obstructive pulmonary disease hospitalized due to an exacerbation: a randomized controlled trial. COPD 2017; 14: 37-42.

13 Borges RC, Carvalho CR. Impact of resistance training in chronic obstructive pulmonary disease patients during periods of acute exacerbation. Arch Phys Med Rehabil 2014; 95: 1638-1645.

14 Giavedoni S, Deans A, McCaughey P, et al. Neuromuscular electrical stimulation prevents muscle function deterioration in exacerbated COPD: a pilot study. Respir Med 2012; 106: 1429-1434.

15 Greulich T, Nell C, Koepke J, et al. Benefits of whole body vibration training in patients hospitalised for COPD exacerbations - a randomized clinical trial. BMC Pulm Med 2014; 14: 60.

16 Burtin C, Clerckx B, Robbeets C, et al. Early exercise in critically ill patients enhances short-term functional recovery. Crit Care Med 2009; 37: 2499-2505.

17 Zanotti E, Felicetti G, Maini M, et al. Peripheral muscle strength training in bed-bound patients with COPD receiving mechanical ventilation: effect of electrical stimulation. Chest 2003; 124: 292-296.

18 Schweickert WD, Pohlman MC, Pohlman AS, et al. Early physical and occupational therapy in mechanically ventilated, critically ill patients: a randomised controlled trial. Lancet 2009; 373: 1874-1882.

19 Nydahl P, Sricharoenchai T, Chandra S, et al. Safety of patient mobilization and rehabilitation in the ICU: systematic review with meta-analysis. Ann Am Thorac Soc 2017; 14: 766-777.

20 Greening NJ, Williams JE, Hussain SF, et al. An early rehabilitation intervention to enhance recovery during hospital admission for an exacerbation of chronic respiratory disease: randomised controlled trial. BMJ 2014; 349 : g4315.

21 Janssen DJ, Schols JM, Wouters EF, et al. One-year stability of care dependency in patients with advanced chronic organ failure. J Am Med Dir Assoc 2014; 15: 127-132.

22 Puhan MA, Gimeno-Santos E, Cates CJ, et al. Pulmonary rehabilitation following exacerbations of chronic obstructive pulmonary disease. Cochrane Database Syst Rev 2016; 12: CD005305.

23 Cox NS, Oliveira CC, Lahham A, et al. Pulmonary rehabilitation referral and participation are commonly influenced by environment, knowledge, and beliefs about consequences: a systematic review using the Theoretical Domains Framework. J Physiother 2017; 63: 84-93. 
24 Rochester CL, Vogiatzis I, Holland AE, et al. An Official American Thoracic Society/European Respiratory Society Policy Statement: Enhancing Implementation, Use, and Delivery of Pulmonary Rehabilitation. Am J Respir Crit Care Med 2015; 192: 1373-1386.

25 Spruit MA, Pitta F, Garvey C, et al. Differences in content and organisational aspects of pulmonary rehabilitation programmes. Eur Respir J 2014; 43: 1326-1337.

26 Jones SE, Green SA, Clark AL, et al. Pulmonary rehabilitation following hospitalisation for acute exacerbation of COPD: referrals, uptake and adherence. Thorax 2014; 69: 181-182.

Copyright @ERS 2018

From the authors:

We thank M.A. Spruit and colleagues for their questions about our decisions regarding initiating pulmonary rehabilitation in patients with chronic obstructive pulmonary disease (COPD) exacerbations. Their main objection is the recommendation against initiating pulmonary rehabilitation during the patient's hospitalisation. We agree that the recommendation was based primarily on the finding of increased mortality (6-min walking test had statistically significant improvement and hospital readmission had non-statistically significant improvement) and that the trial led by GreENING et al. [1] contributed 389 out of 415 patients. We further agree that there is uncertainty about whether or not inpatient-initiated pulmonary rehabilitation is associated with increased mortality, particularly since the mortality difference emerged more than 5 months after hospital discharge in the study by GrEENING et al. [1] and that the per protocol analysis in this study found no difference in mortality among those who actually received pulmonary rehabilitation versus the control group.

However, we believe the guideline panel's judgment is justified. Faced with the decision about whether to recommend for or against an intervention for which the only major clinical trial found increased mortality and no reduction in the re-exacerbation rate, and only a few subsequent analyses raised doubt about the finding, we elected to minimise the potential for harm by recommending against the intervention until further evidence becomes available to revisit the decision. When combined with a recommendation in favour of pulmonary rehabilitation within 3 weeks of discharge, the net effect of the recommendations is a mere delay of days to weeks in the interest patient safety.

M.A. Spruit and colleagues cite the results of a 2016 Cochrane systematic review and meta-analysis to support their concerns [2]. However, they fail to acknowledge that the Cochrane report also found substantial evidence of heterogeneity of treatment effects on hospital readmissions and mortality, and that the "reasons for diverse effects ... are not fully clear. Further studies should explore whether the extent of the rehabilitation program and the organization of such programs within specific healthcare systems (e.g. within the rehabilitation setting versus embedded in the continuum of care from hospital to home to outpatient care) determines the effects of rehabilitation after COPD exacerbations."

We agree with these conclusions in the Cochrane report and, in the European Respiratory Society/ American Thoracic Society guideline [3], we advocated for further research: patients, clinicians and other decision-makers need adequately powered, well-designed randomised clinical trials to more clearly delineate the role of pulmonary rehabilitation in the peri-exacerbation period.

Kevin C. Wilson ${ }^{1}$, Jerry A. Krishnan ${ }^{2}$, Pawel Sliwinski ${ }^{3}$, Gerard J. Criner ${ }^{4}$, Marc Miravitlles $\oplus^{5}$, John R. Hurst ${ }^{6}$, Peter M.A. Calverley ${ }^{7}$, Richard K. Albert ${ }^{8}$, David Rigau' ${ }^{9}$, Thomy Tonia ${ }^{10}$, Jørgen Vestbo ${ }^{11}$, Alberto Papi $\oplus^{12}$, Klaus F. Rabe ${ }^{13}$, Antonio Anzueto ${ }^{14}$ and Jadwiga A. Wedzicha ${ }^{15}$

${ }^{1}$ Dept of Medicine, Boston University School of Medicine, Boston, MA, USA. ${ }^{2}$ University of Illinois Hospital and Health Sciences System, Chicago, IL, USA; co-chair, representing the American Thoracic Society. ${ }^{3} 2$ nd Dept of Respiratory Medicine, Institute of Tuberculosis and Lung Diseases, Warsaw, Poland. ${ }^{4}$ Dept of Thoracic Medicine and Surgery, Lewis Katz School of Medicine at Temple University, Philadelphia, PA, USA. ${ }^{5}$ Pneumology Dept, Hospital Universitari Vall d'Hebron, CIBER de Enferlmedades Respiratorias (CIBERES), Barcelona, Spain. ${ }^{6}$ UCL Respiratory, University College London, London, UK. ${ }^{7}$ Institute of Ageing and Chronic Disease, University of Liverpool, Liverpool, UK. ${ }^{8}$ Dept of Medicine, University of Colorado, Denver, Aurora, CO, USA. ${ }^{9}$ Iberoamerican Cochrane Center, Barcelona, Spain.

${ }^{10}$ Institute of Social and Preventive Medicine, University of Bern, Bern, Switzerland. ${ }^{11}$ Division of Infection,

@ERSpublications

Guideline recommendation against the initiation of pulmonary rehabilitation during hospitalisation was justified http://ow.ly/ve2L30hiqop

Cite this article as: Wilson KC, Krishnan JA, Sliwinski P, et al. Pulmonary rehabilitation for patients with COPD during and after an exacerbation-related hospitalisation: back to the future? Eur Respir J 2018; 51: 1702577 [https://doi.org/10.1183/13993003.02577-2017]. 
Immunology and Respiratory Medicine, The University of Manchester and Manchester University NHS Foundation Trust, Manchester, UK. ${ }^{12}$ Respiratory Medicine, Dept of Medical Sciences, University of Ferrara, Ferrara, Italy. ${ }^{13}$ Dept of Internal Medicine, Christian-Albrechts University, Kiel and LungenClinic Grosshansdorf, Airway Research Centre North, German Centre for Lung Research, Grosshansdorf, Germany. ${ }^{14}$ University of Texas Health Science Center and South Texas Veterans Health Care System, San Antonio, TX, USA. ${ }^{15}$ Airways Disease Section, National Heart and Lung Institute, Imperial College London, UK; co-chair, representing the European Respiratory Society.

Correspondence: Kevin C. Wilson, Dept of Medicine, Boston University School of Medicine, 10 Whitney Ave, Westwood, Boston, 02090 MA, USA. E-mail: kwilson@thoracic.org

Received: Dec 112017 | Accepted: Dec 122017

Conflict of interest: D. Rigau and T. Tonia act as methodologists for the European Respiratory Society. All other disclosures can be found alongside this article at erj.ersjournals.com

\section{References}

1 Greening NJ, Williams JE, Hussain SF, et al. An early rehabilitation intervention to enhance recovery during hospital admission for an exacerbation of chronic respiratory disease: randomized controlled trial. BMJ 2014; 8: 349 .

2 Puhan MA, Gimeno-Santos E, Cates CJ, et al. Pulmonary rehabilitation following exacerbations of chronic obstructive pulmonary disease. Cochrane Database Syst Rev 2016; 12: CD005305.

3 Wedzicha JA, Miravitlles M, Hurst JR, et al. Management of COPD exacerbations: a European Respiratory Society/ American Thoracic Society guideline. Eur Respir J 2017; 49: 1600791. 